\title{
TU/e emonownen

\section{Improvement of the interfacial Dzyaloshinskii-Moriya interaction by introducing a Ta buffer layer}

\section{Citation for published version (APA):}

Kim, N. H., Han, D. S., Jung, J., Cho, J., Kim, J. S., Swagten, H. J. M., \& You, C. Y. (2015). Improvement of the interfacial Dzyaloshinskii-Moriya interaction by introducing a Ta buffer layer. Applied Physics Letters, 107(14), 14. [142408]. https://doi.org/10.1063/1.4932550

DOI:

$10.1063 / 1.4932550$

Document status and date:

Published: 05/10/2015

\section{Document Version:}

Publisher's PDF, also known as Version of Record (includes final page, issue and volume numbers)

\section{Please check the document version of this publication:}

- A submitted manuscript is the version of the article upon submission and before peer-review. There can be important differences between the submitted version and the official published version of record. People interested in the research are advised to contact the author for the final version of the publication, or visit the $\mathrm{DOI}$ to the publisher's website.

- The final author version and the galley proof are versions of the publication after peer review.

- The final published version features the final layout of the paper including the volume, issue and page numbers.

Link to publication

\section{General rights}

Copyright and moral rights for the publications made accessible in the public portal are retained by the authors and/or other copyright owners and it is a condition of accessing publications that users recognise and abide by the legal requirements associated with these rights.

- Users may download and print one copy of any publication from the public portal for the purpose of private study or research.

- You may not further distribute the material or use it for any profit-making activity or commercial gain

- You may freely distribute the URL identifying the publication in the public portal.

If the publication is distributed under the terms of Article 25fa of the Dutch Copyright Act, indicated by the "Taverne" license above, please follow below link for the End User Agreement:

www.tue.nl/taverne

Take down policy

If you believe that this document breaches copyright please contact us at:

openaccess@tue.nl

providing details and we will investigate your claim. 


\section{AIP/Apolied Physises \\ Letters}

\section{Improvement of the interfacial Dzyaloshinskii-Moriya interaction by introducing a Ta}

buffer layer

Nam-Hui Kim, Dong-Soo Han, Jinyong Jung, Jaehun Cho, June-Seo Kim, Henk J. M. Swagten, and Chun-Yeol You

Citation: Applied Physics Letters 107, 142408 (2015); doi: 10.1063/1.4932550

View online: http://dx.doi.org/10.1063/1.4932550

View Table of Contents: http://scitation.aip.org/content/aip/journal/apl/107/14?ver=pdfcov

Published by the AIP Publishing

\section{Articles you may be interested in}

Interfacial Dzyaloshinskii-Moriya interaction, surface anisotropy energy, and spin pumping at spin orbit coupled Ir/Co interface

Appl. Phys. Lett. 108, 142406 (2016); 10.1063/1.4945685

Influence of interfacial Dzyaloshinskii-Moriya interaction on the parametric amplification of spin waves

Appl. Phys. Lett. 107, 112402 (2015); 10.1063/1.4931089

In-plane angular dependence of the spin-wave nonreciprocity of an ultrathin film with Dzyaloshinskii-Moriya interaction

Appl. Phys. Lett. 107, 022402 (2015); 10.1063/1.4926862

Interface boundary conditions for dynamic magnetization and spin wave dynamics in a ferromagnetic layer with the interface Dzyaloshinskii-Moriya interaction

J. Appl. Phys. 115, 233902 (2014); 10.1063/1.4883181

Chiral magnetization textures stabilized by the Dzyaloshinskii-Moriya interaction during spin-orbit torque switching

Appl. Phys. Lett. 104, 092403 (2014); 10.1063/1.4867199

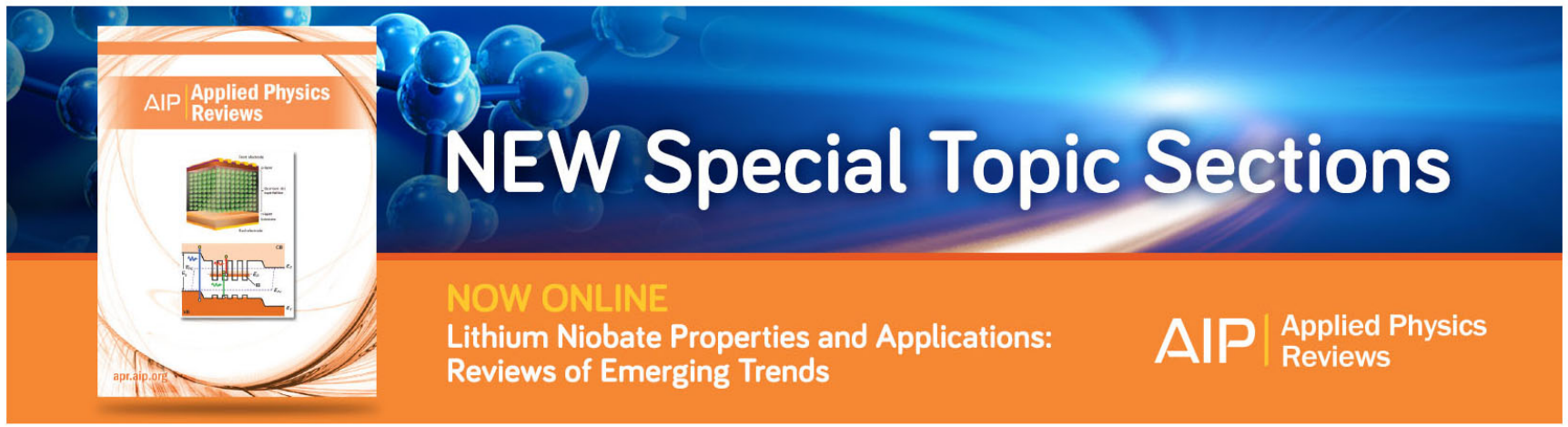




\title{
Improvement of the interfacial Dzyaloshinskii-Moriya interaction by introducing a Ta buffer layer
}

\author{
Nam-Hui Kim, ${ }^{1}$ Dong-Soo Han, ${ }^{2}$ Jinyong Jung, ${ }^{1}$ Jaehun Cho, ${ }^{1}$ June-Seo Kim,,a) \\ Henk J. M. Swagten, ${ }^{2}$ and Chun-Yeol You ${ }^{1, b)}$ \\ ${ }^{1}$ Department of Physics, Inha University, Incheon 402-751, South Korea \\ ${ }^{2}$ Department of Applied Physics, Center for NanoMaterials, Eindhoven University of Technology, PO Box 513 , \\ 5600 MB Eindhoven, The Netherlands
}

(Received 27 July 2015; accepted 24 September 2015; published online 9 October 2015)

\begin{abstract}
We report systematic measurements of the interfacial Dzyaloshinskii-Moriya interaction (iDMI) by employing Brillouin light scattering in $\mathrm{Pt} / \mathrm{Co} / \mathrm{AlO}_{\mathrm{x}}$ and $\mathrm{Ta} / \mathrm{Pt} / \mathrm{Co} / \mathrm{AlO}_{\mathrm{x}}$ structures. By introducing a tantalum buffer layer, the saturation magnetization and the interfacial perpendicular magnetic anisotropy are significantly improved due to the better interface between heavy metal and ferromagnetic layer. From the frequency shift between Stokes- and anti-Stokes spin-waves, we successively obtain considerably larger iDM energy densities $\left(D_{\max }=1.65 \pm 0.13 \mathrm{~mJ} / \mathrm{m}^{2}\right.$ at $\left.t_{\mathrm{Co}}=1.35 \mathrm{~nm}\right)$ upon adding the Ta buffer layer, despite the nominally identical interface materials. Moreover, the energy density shows an inverse proportionality with the Co layer thickness, which is the critical clue that the observed iDMI is indeed originating from the interface between the Pt and Co layers. (C) 2015 AIP Publishing LLC. [http://dx.doi.org/10.1063/1.4932550]
\end{abstract}

In a system with structural inversion asymmetry, spinorbit coupling at the interfaces introduces an additional asymmetric exchange interaction, which is the so-called interfacial Dzyaloshinskii-Moriya interaction (iDMI). ${ }^{1-4}$ This interfacial nature has recently been experimentally examined because of its massive potential to explore radically different magnetic memory and logic devices based on chiral domain wall dynamics ${ }^{5-8}$ and skyrmions, ${ }^{9-11}$ which are topologically protected vortex- or hedgehog-like spin structures. In order to drive these state-of-the-art technologies on a commercial scale, a larger iDM energy density is strongly required. Recently, it was demonstrated by Brillouin Light Scattering (BLS) that in inversion symmetry broken multilayers $\left(\mathrm{Pt} / \mathrm{Co} / \mathrm{AlO}_{\mathrm{x}}\right.$ and $\left.\mathrm{Pt} / \mathrm{CoFeB} / \mathrm{AlO}_{\mathrm{x}}\right)$, the iDM energy density is inversely proportional to the ferromagnetic layer thickness. ${ }^{12}$ It indicates that the iDMI is purely originating at the interfaces, as also indicated by recent ab-initio calculations. ${ }^{13}$ Moreover, the use of a proper buffer layer can help us to improve the iDMI by introducing an reduced roughness of the interfaces. To increase the interface quality, a buffer layer, especially tantalum (Ta) is widely used in various research fields in magnetism such as perpendicular magnetic recording media composed of $\mathrm{Co} / \mathrm{Pt}$, $\mathrm{Co} / \mathrm{Pd}$, multilayer system. Since a Ta seed layer can introduce an atomically smooth interface at $\mathrm{Pt} / \mathrm{Co}$, a strong interfacial perpendicular magnetic anisotropy (iPMA) can be achieved by the high strain effect, ${ }^{14-16}$ and so on. ${ }^{17-19}$

In this letter, we experimentally demonstrate that a $\mathrm{Ta}$ buffer layer is able to enhance the iPMA and also the iDMI in $\mathrm{Pt} / \mathrm{Co} / \mathrm{AlO}_{\mathrm{x}}$ magnetic multilayer system, which is believed to originate from a better $\mathrm{Pt} / \mathrm{Co}$ interfaces. In order to investigate these interfacial phenomena, we perform BLS measurements, which is sensitive to the surface spin wave (SW)

\footnotetext{
a)spin2mtj@gmail.com

b) cyyou@inha.ac.kr
}

excitations. From systematic measurements, we observe the frequency differences between two independent propagating SWs, since these are straightforwardly proportional to the iDM energy density. By introducing a Ta buffer layer, significantly improved iDM energies are clearly observed. Again, the inverse proportionalities of iDMI and iPMA give us strong evidence for the pure interfacial origin of these phenomena.

Our sample consists of Ta $(4 \mathrm{~nm}) / \mathrm{Pt}(4 \mathrm{~nm}) / \mathrm{Co}\left(t_{\mathrm{Co}} \mathrm{nm}\right) /$ $\mathrm{AlO}_{\mathrm{x}}(2 \mathrm{~nm})$ on a thermally oxidized $\mathrm{Si}$-wafer. All the layers are deposited by using a magnetron sputtering system, and especially, the Co layer is wedged in the range of $1.30-1.80 \mathrm{~nm}$. As depicted in Fig. 1(a), BLS measurements are carried out with strong in-plane magnetic ( $x$-direction in experimentally coordinate system) applied fields up to $H_{\mathrm{ex}}=0.9 \mathrm{~T}$. The BLS spectra are observed by using a $(3+3)$ multi-pass tandem Fabry-Pérot interferometer, and a $p$-polarized LASER (300 $\mathrm{mW}$ power and $532 \mathrm{~nm}$ wavelength) is used as a light source. The back-scattered light is focused from the sample, and the $s$-polarized lights are passed through the interferometer and collected by the photomultiplier tubes. ${ }^{12,20,21}$ In general, BLS spectra representing Damon-Eshbach surface modes are composed of two propagating SWs signals, the so-called Stokes and anti-Stokes peaks from two different interfaces or surfaces. If the interfaces are identical, two peaks should be located at the same frequency. For non-identical cases, the frequency difference between two propagating SWs exists, which is, e.g., seen in Figure 1(b) showing the BLS spectrum for $\mathrm{Ta} / \mathrm{Pt} / \mathrm{Co} / \mathrm{AlO}_{\mathrm{x}}$ with a huge frequency difference $(\Delta f=2.56 \mathrm{GHz})$. In line with earlier results, ${ }^{12}$ it reflects that our inversion symmetry broken system contains a significant iDMI. ${ }^{12,22-24}$ More details on the measurements and interpretation are explained in Ref. 12.

As a versatile tool, BLS is able to determine other magnetic properties as well, namely, the anisotropy energy, the 
(a)
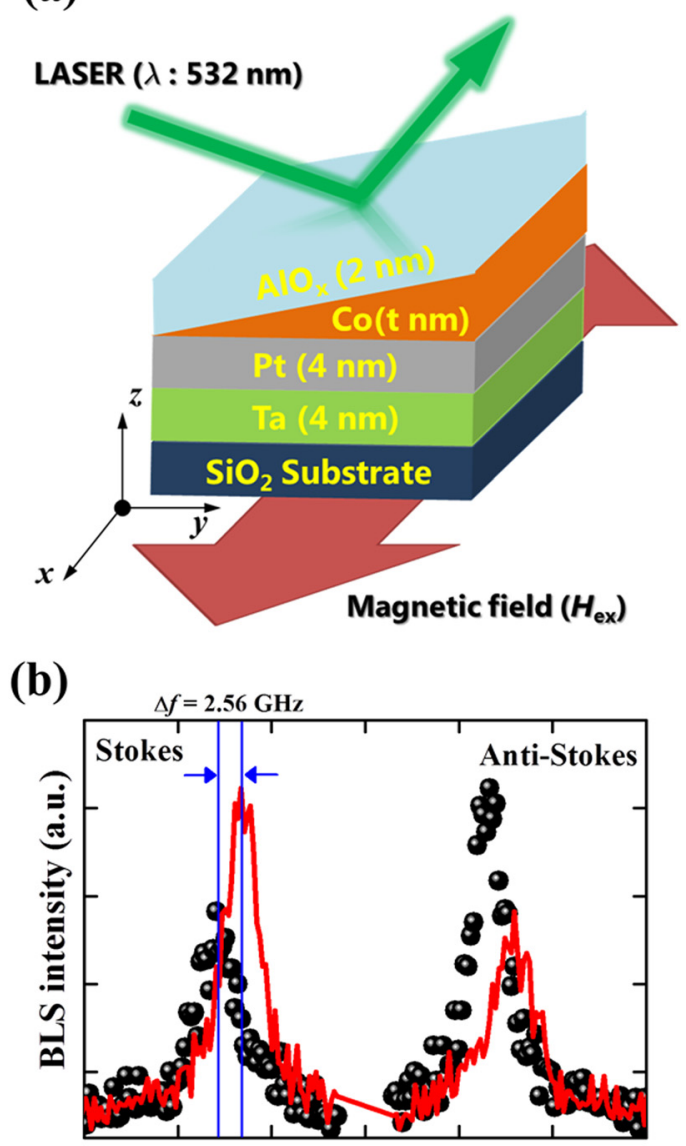

Frequency $(\mathbf{G H z})$

FIG. 1. (a) Schematic for the sample structure and BLS measurement. The sample consist of $\mathrm{SiO}_{2}$ sub./Ta/Pt/Co/AlO $(1.35-1.80 \mathrm{~nm})$. (b) The spin wave spectrum obtained from 1.35 -nm thick Co layer with an in-plane magnetic field $H_{\text {ext }}=0.79 \mathrm{~T}$. The incident angle is fixed at $\theta=45^{\circ}$ (corresponding to $k_{\mathrm{y}}=0.0167 \mathrm{~nm}^{-1}$ ). In order to identify the frequency difference $(\Delta f)$ between Stokes and anti-Stokes, the mirrored curve (red solid lines) is overlapped in the spectrum.

saturation magnetization, and the exchange stiffness constant with the various types of the propagating SWs. ${ }^{20,21,25}$ From BLS measurements, we deduce the effective saturation magnetization value $\left(M_{\text {eff }}=M_{\mathrm{s}}-\frac{4 K_{\mathrm{s}}}{\mu_{0} M_{\mathrm{s} \mathrm{t}_{\mathrm{c}}}}\right)$ from the measured $\mathrm{SW}$ frequency $\left(f_{\mathrm{SW}}=\frac{\gamma}{2 \pi} \sqrt{H_{\mathrm{ex}}\left(H_{\mathrm{ex}}-\mu_{0} M_{\mathrm{S}}+\frac{4 K_{\mathrm{s}}}{M_{\mathrm{s}} t_{\mathrm{C}}}\right)}\right)$, where $\gamma, H_{\mathrm{ex}}, M_{\mathrm{s}}$, and $K_{\mathrm{s}}$ are the gyromagnetic ratio, the applied magnetic field, the saturation magnetization and the surface anisotropy, respectively. ${ }^{26}$ In order to extract the change of magnetic anisotropy energy from the measured SWs frequencies in each Co thickness, we used the effective uniaxial anisotropy energy $K_{\text {eff }}$ corresponding to the surface anisotropy $\left(K_{\mathrm{s}}\right)$ and the volume anisotropy $\left(-\frac{1}{2} \mu_{0} M_{\mathrm{s}}^{2}\right)$ given by

$$
K_{\mathrm{eff}} \times t_{\mathrm{Co}}=2 K_{\mathrm{s}}-\frac{1}{2} \mu_{0} M_{\mathrm{s}}^{2} \times t_{\mathrm{Co}} .
$$

Figure 2 indicates that we observe the linear dependences of $K_{\text {eff }} \times t_{\text {Co }}$ versus $t_{\text {Co }}$ with (black squares) and without a Ta buffer (red circles). As shown in Fig. 2, the extrapolated crossing with the y-axis $K_{\mathrm{s}}$ and the slope $\left(-\frac{1}{2} \mu_{0} M_{\mathrm{s}}^{2}\right)$ are significantly

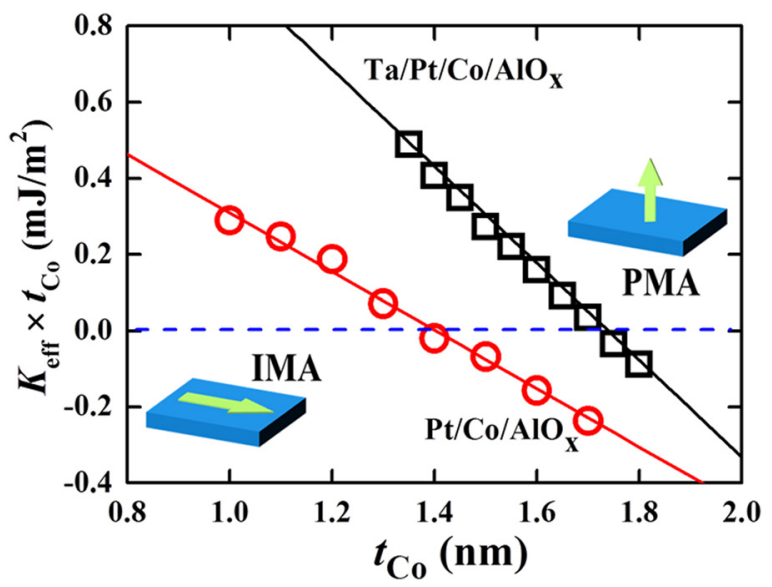

FIG. 2. The $K_{\text {eff }} \times t_{\text {Co }} v s$. $t_{\text {Co }}$ plot with a linear fitting. Above $t_{\text {Co }}>1.73 \mathrm{~nm}$ (with a Ta buffer layer, black squares) and $t_{\mathrm{Co}}>1.42 \mathrm{~nm}$ (without a Ta buffer layer, red circles), the effective uniaxial anisotropy becomes negative, which means the easy axis of the sample is in-plane.

enhanced due to the Ta buffer layer. Consequently, the addition of Ta leads to enhance the interface quality of $\mathrm{Pt} / \mathrm{Co}$. We will discuss the quantitative changes of $K_{\mathrm{s}}\left(1.10 \mathrm{~mJ} / \mathrm{m}^{2}\right)$ and $M_{\mathrm{s}}$ $(1423 \mathrm{kA} / \mathrm{m})$ with Ta buffer layer later on in this paper.

To extract the iDM energy density by using BLS, the first step is observing the frequency differences $(\Delta f)$ between Stokes and anti-Stokes peaks in BLS spectra. In Figure 3(a), $\Delta f$ with a buffer (black squares) and without a buffer (red

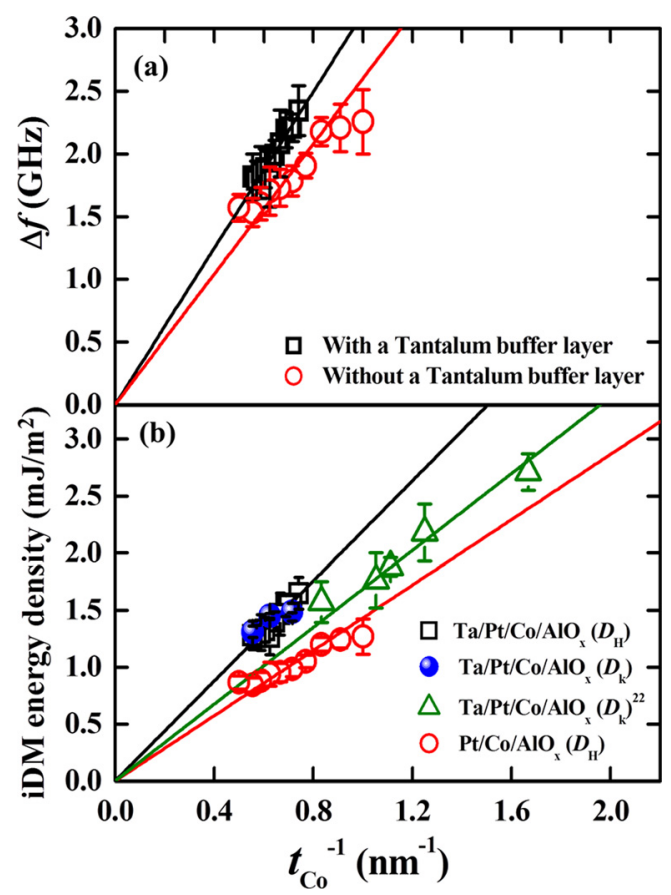

FIG. 3. (a) $\Delta f$ as a function of $t_{\mathrm{Co}}^{-1}$. Black squares and red squares indicate $\mathrm{Ta} / \mathrm{Pt} / \mathrm{Co} / \mathrm{AlO}_{\mathrm{x}}$ and $\mathrm{Pt} / \mathrm{Co} / \mathrm{AlO}_{\mathrm{x}},{ }^{12}$ respectively. For these measurements, the incident angle is fixed at $\theta=45^{\circ}$, which corresponds to the $k_{\mathrm{y}}=0.0167 \mathrm{~nm}^{-1}$. (b) The iDM energy density as a function of $t_{\mathrm{Co}}^{-1}$ for the two measurement methods. Black squares and red circles indicate the iDM energy density measured by external magnetic field dependence $\left(D_{\mathrm{H}}\right)$ for with (black squares)/without Ta buffer layer (red circles) cases. The blue spheres stand for SW wave-vector dependence results $\left(D_{\mathrm{k}}\right) . D_{\mathrm{k}}$ are determined from the linear fit of Eq. (2) to the $k_{\mathrm{y}}$ for each thickness $\left(t_{\mathrm{Co}}=1.4\right.$, 1.6 , and $1.8 \mathrm{~nm}$ ). Consequently, black squares, blue spheres and green triangles (Ref. 22) show clearly more improved iDM energy densities, when a Ta is used for buffer layer. 
circles) $)^{12}$ are shown as a function of $t_{\mathrm{Co}}{ }^{-1}$. Here, $\Delta f$ for each thickness $t_{\mathrm{Co}}$ are determined from the field dependent measurements (from 0 to $0.9 \mathrm{~T}$ ), and the measured $\Delta f$ should be a constant for all magnetic fields (Ref. 12). Therefore, in Fig. 3 , symbols and error bars indicate the averaged values and the corresponding standard deviations of the $\Delta f$, respectively. We plot the $\Delta f$ as a function of $t_{\mathrm{Co}}^{-1}$ and it clearly shows the inverse proportionality with $t_{\mathrm{Co}}$. The physical meaning of the inverse proportionality is that a bulk contribution screens the interface effects with increasing $t_{\mathrm{Co}}$. In various magnetic systems, the inverse proportionality to the ferromagnetic layer thickness is a signature of the interface effects such as interface PMA,${ }^{27}$ exchange bias, ${ }^{28}$ the effective field of the interlayer exchange coupling, ${ }^{29}$ and so on. ${ }^{30}$ Apart from the observed interfacial nature of $\Delta f$, we found that $\Delta f$ with Ta buffer layers is much larger than $\Delta f$ without Ta buffer layers, since it is directly linked to the iDM energy density, which is given by

$$
\Delta f=\frac{2 \gamma D}{\pi M_{\mathrm{s}}} k_{\mathrm{y}},
$$

where $k_{\mathrm{y}}$ and $D$ are the propagating SW $k$-vector along the $y$ direction and the iDM energy density, respectively. The SW vector is fixed at $k_{\mathrm{y}}=0.0167 \mathrm{~nm}^{-1}$ for the field dependent measurements, and it is varied from 0.01 to $0.02 \mathrm{~nm}^{-1}$ for the SW wave-vector dependent measurements.

Figure 3(b) shows iDM energy density deduced from Eq. (2) as a function of $t_{\mathrm{Co}}^{-1}$ with and without a Ta buffer layer from the magnetic field dependent measurement $\left(D_{\mathrm{H}}\right)$. We also included the SW wave-vector dependent results $\left(D_{\mathrm{k}}\right)$ as blue spheres in Fig. 3(b) for selected Co thicknesses (1.4, 1.6, and $1.8 \mathrm{~nm}$ ), which we obtain from varying the propagating spin-wave $k$-vector $\left(0.01 \mathrm{~nm}^{-1}<k_{\mathrm{y}}<0.02 \mathrm{~nm}^{-1}\right)$. The excellent agreement between two measurement results $\left(D_{\mathrm{H}}\right.$ and $\left.D_{\mathrm{k}}\right)$ implies that our results are independent from possible artifacts as already discussed in Ref. 12.

There are two main issues in this study which we would like to discuss in more detail. First, the iDM energy density with a Ta buffer layer $\left(D_{\mathrm{H}}=1.56 \mathrm{~mJ} / \mathrm{m}^{2}\right)$ is noticeably enhanced approximately $58 \%$ compared to the absence of Ta $\left(D_{\mathrm{H}}=0.98 \mathrm{~mJ} / \mathrm{m}^{2}\right)$ on the same thickness $\left(t_{\mathrm{Co}}=1.4 \mathrm{~nm}\right)$. In Ref. 22, the authors have used the same buffer layer and measured iDM energy density by using BLS. The thickness dependent iDM energy densities from Ref. 22 are depicted in Fig. 3(b) (green triangles) and their measured iDM energy densities with a $\mathrm{Ta}$ buffer are also reasonably large. Consequently, their results can support our data that a Ta buffer layer is able to improve the iDMI. Second, the iPMA and $M_{\mathrm{s}}$ values are also enhanced by $103 \%$ and $29 \%$, respectively, by adding the Ta buffer layer. In order to have a strong interfacial surface anisotropy at the interface between $\mathrm{Co}$ and Pt, FCC (111) orientation to induce a high strain effect is necessary. Previous results ${ }^{14-16}$ can clearly support our data that a Ta seed layer can introduce an atomically smooth interface at $\mathrm{Pt} / \mathrm{Co}$ and then a strong interfacial PMA can be achieved by the high strain effect. Therefore, a Ta buffer decreases the interfacial roughness and then the interface has a strong magnetic anisotropy. As a result, better interface quality will provide stronger spin-orbit coupling, which is the source not only for iDM interaction, but also for the iPMA and spin polarization of the Pt layer.

In Fig. 3(a), the slopes of $\Delta f$ values for with and without Ta buffer are similar to each other. However, Fig. 3(b) shows that the slopes of the iDM energy densities are quite different. It is not a surprising result, because $M_{\mathrm{s}}$ is closely related with the exchange stiffness constant $A_{\mathrm{ex}}$, and $D$ should be proportional to $A_{\text {ex }}{ }^{31}$ Therefore, we are able to highlight that the case of a Ta buffer layer gives us a larger $M_{\mathrm{s}}(=1423 \mathrm{kA} /$ $\mathrm{m})$, which is quite close to the bulk value of $\mathrm{Co}^{32}$ There are two possible scenarios for the large $M_{\mathrm{s}}$ close to bulk Co. First, the improved the interface between Pt and Co layers makes the Co better defined without much intermixing, which should enhance $M_{\mathrm{s}}$ towards the bulk. The second scenario is the proximity effect of the Pt. ${ }^{33}$ It is well known that $\mathrm{Pt}$ is easily spin polarized and becomes ferromagnet when it is adjacent to the ferromagnetic layer due to the strong spinorbit coupling and band hybridizations. ${ }^{34-36}$ Therefore, the spin polarized ferromagnetic Pt may contribute to the measured $M_{\mathrm{s}}$. Without further analysis on systems with a systematic variation of the Pt layer thickness as well (which is beyond the scope of this paper concentrating on iDMI), we are not able to discriminate between the two scenarios.

Finally, we discuss the role of a Ta buffer layer in view of skyrmion formation conditions. ${ }^{8,37}$ The skyrmion phase can be formed when the domain wall energy density, $\sigma=4 \sqrt{A_{\text {ex }} K_{\text {eff }}}-\pi D$, becomes negative, from which we obtain the critical iDMI energy density, $D_{\text {cri }}=4 / \pi$ $\sqrt{A_{\text {ex }} K_{\text {eff }}}$. In our study, we found averagely $58 \%$ enhancement of $D$; however, $K_{\mathrm{s}}$ also increases by about $103 \%$. In addition, we speculated on an increase of $A_{\text {ex }}$ based on the relation with $M_{\mathrm{s}}$. Even though we enhanced $D$ with a Ta buffer layer, it leads to the enhancement of $K_{\mathrm{s}}$ and $A_{\text {ex }}$, and causes an increase of $D_{\text {cri. }}$ Therefore, independent control of $D, K_{\mathrm{s}}$, and $A_{\mathrm{ex}}$ is necessary in order to satisfy the condition for skyrmion formation.

In conclusion, from BLS measurement in $\mathrm{Pt} / \mathrm{Co} / \mathrm{AlO}_{\mathrm{x}}$ and $\mathrm{Ta} / \mathrm{Pt} / \mathrm{Co} / \mathrm{AlO}_{\mathrm{x}}$, we obtain that the Ta-buffer significantly enhances the surface magnetic anisotropy $K_{\mathrm{s}}$, the saturation magnetization $M_{\mathrm{s}}$, and the interfacial DzyaloshinskiiMoriya interaction (iDMI). Finally, we emphasize that by engineering the interface quality by introducing a proper $\mathrm{Ta}$ buffer layer, we achieved a $58 \%$ enhancement of $D$, despite of the nominally identical interface materials. It implies that there is ample room for improving $D$ by interfacial and structural engineering.

This work was supported by the research programme of the Foundation for Fundamental Research on Matter (FOM), which is part of the Netherlands Organisation for Scientific Research (NWO), and the National Research Foundation of Korea (Grant Nos. 2015M3D1A1035354, 2015M2A2A6021171, and 2013R1A1A2011936).

\footnotetext{
${ }^{1}$ A. Fert and P. M. Levy, Phys. Rev. Lett. 44, 1538-1541 (1980).

${ }^{2}$ A. Fert, Mater. Sci. Forum 59, 439-480 (1991).

${ }^{3}$ A. Thiaville, S. Rohart, É. Jué, V. Cros, and A. Fert, EPL 100, 57002 (2012).

${ }^{4}$ R. Yanes, J. Jackson, L. Udvardi, L. Szunyogh, and U. Nowak, Phys. Rev. Lett. 111, 217202 (2013).
} 
${ }^{5}$ S. G. Je, D.-H. Kim, S.-C. Yoo, B.-C. Min, K.-J. Lee, and S.-B. Choe, Phys. Rev. B 88, 214401 (2013).

${ }^{6}$ K. S. Ryu, L. Thomas, S. H. Yang, and S. Parkin, Nat. Nanotechnol. 8, 527-533 (2013).

${ }^{7}$ S. Pizzini, J. Vogel, S. Rohart, L. D. Buda-Prejbeanu, E. Jué, O. Boulle, I. M. Miron, C. K. Safeer, S. Auffret, G. Gaudin, and A. Thiaville, Phys. Rev. Lett. 113, 047203 (2014).

${ }^{8}$ A. Hrabec, N. A. Porter, A. Wells, M. J. Benitez, G. Burnell, S. McVitie, D. McGrouther, T. A. Moore, and C. H. Marrows, Phys. Rev. B 90, 020402 (2014).

${ }^{9}$ J. Sampaio, V. Cros, S. Rohart, A. Thiaville, and A. Fert, Nat. Nanotechnol. 8, 839-844 (2013).

${ }^{10}$ A. Fert, V. Cros, and J. Sampaio, Nat. Nanotechnol. 8, 152-156 (2013).

${ }^{11}$ S. Rohart and A. Thiaville, Phys. Rev. B 88, 184422 (2013).

${ }^{12}$ J. Cho, N.-H. Kim, S. Lee, J.-S. Kim, R. Lavrijsen, A. Solignac, Y. Yin, D.-S. Han, N. J. J. Hoof, H. J. M. Swagten, B. Koopmans, and C.-Y. You, Nat. Commun. 6, 7635 (2015).

${ }^{13}$ H. Yang, S. Rohart, A. Thiaville, A. Fert, and M. Chshiev, in APS March Meeting 2015, San Antonio, Texas, USA, 2 March-6 March 2015, Bulletin of the American Physical Society, 60 (2015).

${ }^{14}$ W. Peng, O. Keitel, R. H. Victora, E. Koparal, and J. H. Judy, IEEE Trans. Magn. 36, 2390 (2000).

${ }^{15}$ J. M. Shaw, H. T. Nembach, T. J. Silva, S. E. Russek, R. Geiss, C. Jones, N. Clark, T. Leo, and D. J. Smith, Phys. Rev. B 80, 184419 (2009).

${ }^{16}$ S. Kim, S. Lee, J. Kim, J. Kang, and J. Hong, J. Appl. Phys. 109, $07 B 766$ (1990).

${ }^{17}$ M. Yamanouchi, R. Koizumi, S. Ikeda, H. Sato, K. Mizunuma, K. Miura, H. D. Gan, F. Matsukura, and H. Ohno, J. Appl. Phys. 109, 07 C712 (2011).

${ }^{18}$ A. Lamperti, S.-M. Ahn, B. Ocker, R. Mantovan, and D. Ravelosona, Thin Solid Films 533, 79-82 (2013).

${ }^{19}$ R. Lo Conte, A. Hrabec, A. P. Mihai, T. Schulz, S.-J. Noh, C. H. Marrows, T. A. Moore, and M. Kläui, Appl. Phys. Lett. 105, 122404 (2014).
${ }^{20}$ S.-S. Ha, N.-H. Kim, S. Lee, C.-Y. You, Y. Shiota, T. Maruyama, T. Nozaki, and Y. Suzuki, Appl. Phys. Lett. 96, 142512 (2010).

${ }^{21}$ S.-S. Ha, N.-H. Kim, C.-Y. You, S. Lee, K. Ohta, T. Maruyama, K. Konishi, T. Nozaki, Y. Suzuki, and W. V. Roy, IEEE Trans. Magn. 45, 2527 (2009).

${ }^{22}$ M. Belmeguenai, J.-P. Adam, Y. Roussigné, S. Eimer, T. Devolder, J.-V. Kim, S. M. Cherif, A. Stashkevich, and A. Thiaville, Phys. Rev. B 91, 180405(R) (2015)

${ }^{23}$ K. Di, V. L. Zhang, H. S. Lim, S. C. Ng, M. H. Kuok, X. Qiu, and H. Yang, Appl. Phys. Lett. 106, 052403 (2015).

${ }^{24}$ K. Di, V. L. Zhang, H. S. Lim, S. C. Ng, M. H. Kuok, J. Yu, J. Yoon, X. Qiu, and H. Yang, Phys. Rev. Lett. 114, 047201 (2015).

${ }^{25}$ J. Cho, J. Jung, K.-E. Kim, S.-I. Kim, S.-Y. Park, M.-H. Jung, and C.-Y. You, J. Mag. Mag. Mater. 339, 36 (2013).

${ }^{26}$ J. R. Dutcher, B. Heinrich, J. F. Cochran, D. A. Steigerwald, and W. F. Egelhoff, Jr., J. Appl. Phys. 63, 3464-3466 (1988).

${ }^{27}$ M. T. Johnson, P. J. H. Bloemen, F. J. A. den Broeder, and J. J. de Vries, Rep. Prog. Phys. 59, 1409-1458 (1996).

${ }^{28}$ J. G. Hu, G. J. Jin, and Y. Q. Ma, J. Appl. Phys. 94, 2529-2533 (2003).

${ }^{29}$ G. Binasch, P. Grünberg, F. Saurenbach, and W. Zinn, Phys. Rev. B 39, 4828(R) (1989).

${ }^{30}$ J. Z. Sun, Phys. Rev. B 62, 570-578 (2000).

${ }^{31}$ H. T. Nembach, J. M. Shaw, M. Weiler, E. Jué, and T. J. Silva, Nat. Phys. 11, 825-829 (2015).

${ }^{32}$ R. Lavrijsen, D. M. F. Hartmann, A. van den Brink, Y. Yin, B. Barcones, R. A. Duine, M. A. Verheijen, H. J. M. Swagten, and B. Koopmans, Phys. Rev. B 91, 104414 (2015).

${ }^{33}$ K.-S. Ryu, S.-H. Yang, L. Thomas, and S. S. P. Parkin, Nat. Commun. 5, 3910 (2014).

${ }^{34}$ J. Crangle and W. R. Scott, J. Appl. Phys. 36, 921-927 (1965).

${ }^{35}$ D. G. Stinson and S.-C. Shin, J. Appl. Phys. 67, 4459-4461(1990).

${ }^{36}$ R. H. Victora and J. M. MacLaren, J. Appl. Phys. 69, 5652-5654 (1991).

${ }^{37}$ C.-Y. You and N.-H. Kim, Curr. Appl. Phys. 15(3), 298-301 (2015). 